\title{
Rare Case of Spontaneous Pneumorrhachis in a Young Male with COVID-19
}

\author{
Vikas Reddy $^{1}$ Ankush Jajodia ${ }^{10}$ Sunil Pasricha ${ }^{2}{ }^{(0)}$ \\ ${ }^{1}$ Department of Radiology, Rajiv Gandhi Cancer Institute and \\ Research Centre, Delhi, India \\ 2 Department of Histopathology, Rajiv Gandhi Cancer Institute, Delhi, \\ India \\ ${ }^{3}$ Department of Medical Oncology, Rajiv Gandhi Cancer Institute and \\ Research Centre, Delhi, India \\ Indian J Radiol Imaging 2021;31:761-763.
}

\author{
Sumit Goyal ${ }^{3}$ Sunil Puri ${ }^{1}$
}

\begin{abstract}
Address for correspondence Ankush Jajodia, Department of Radiology, Rajiv Gandhi Cancer Institute and Research Centre, Rohini Sector 5, Delhi 110085, India (e-mail: ankushjaj@gmail.com).
\end{abstract}

\begin{abstract}
Keywords

- pneumomediastinum

- pneumorrhachis

- COVID-19

Here we present a rare case of spontaneous pneumomediastinum complicated with pneumorrhachis (PR) in a young man who is a known case of carcinoma rectosigmoid region. Our young male was diagnosed with novel coronavirus disease 2019 (COVID-19) and remained asymptomatic for any respiratory complaints. Though an association of pneumomediastinum has been reported in COVID 19 patients, to the best of our knowledge, this is the first report of PR in a COVID-19 oncological case. The role of a radiologist is to identify this condition as cases reported earlier may have serious neurological consequences. Pursuing the cause can be a diagnostic challenge but it reaps the clinical benefit in the appropriate management.
\end{abstract}

\section{Introduction}

Pneumomediastinum is called spontaneous when other common causes, such as trauma, surgery, interventions, or chest infections, have been ruled out. ${ }^{1}$ Pneumorrhachis (PR) is the presence of air within the spinal canal, either intra or extradural in location. ${ }^{2}$ It is mostly associated with epidural anesthesia, lumbar puncture, spinal surgery, spinal injury, and traumatic pneumothorax. Nontraumatic spontaneous PR is extremely rare but can occur as a rare complication of spontaneous pneumomediastinum. PR associated with spontaneous pneumomediastinum is generally asymptomatic. $^{3}$ Tsuji et al report instances of occurrence of PR associated with asthma and violent coughing. ${ }^{4}$ The route of entry into the spinal canal is through the neural foramen from the posterior mediastinum due to the absence of a barrier between the posterior mediastinum and epidural space.,

\section{Case Report}

A 22-year-old normotensive nondiabetic male, a follow-up case of rectosigmoid carcinoma, presented with complaints of recurrent bilious vomiting. With a provisional diagnosis of subacute intestinal obstruction (SAIO), a computed tomography (CT) enterography was ordered by the treating physician which revealed ascites with dilated small bowel loops and peritoneal disease. He underwent emergent exploratory laparotomy for SAIO. After uneventful postoperative care, the patient followed-up on a regular outpatient medical oncology facility for the decision on adjuvant treatment. In the Multispecialty tumor board discussion, a course of adjuvant chemotherapy was deemed appropriate as next step in management, before which the patient underwent an reverse-transcription polymerase chain reaction (RT-PCR) test as per institutional protocol. He had no respiratory
DOI https://doi.org/ 10.1055/s-0041-1735920. ISSN 0971-3026.

\footnotetext{
(C) 2021. Indian Radiological Association. All rights reserved. This is an open access article published by Thieme under the terms of the Creative Commons Attribution-NonDerivative-NonCommercial-License, permitting copying and reproduction so long as the original work is given appropriate credit. Contents may not be used for commercial purposes, or adapted, remixed, transformed or built upon. (https://creativecommons.org/ licenses/by-nc-nd/4.0/) Thieme Medical and Scientific Publishers Pvt. Ltd., A-12, 2nd Floor, Sector 2, Noida-201301 UP, India
} 


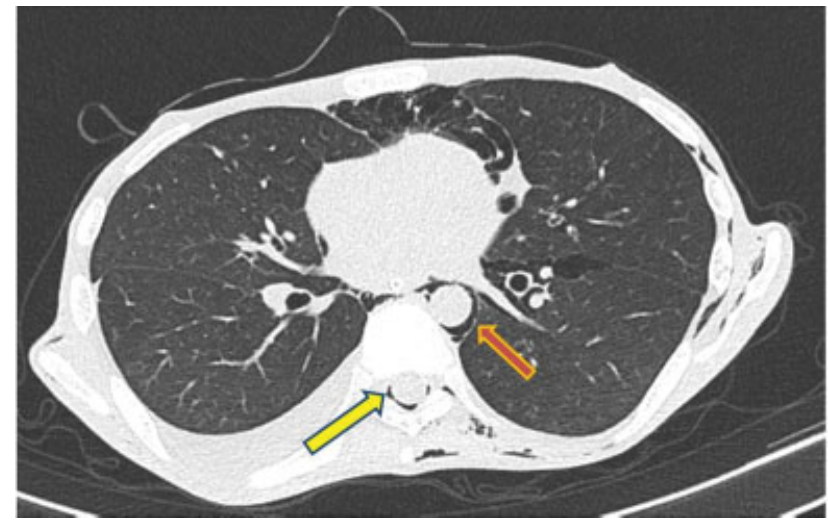

Fig. 1 Axial CT image shows few tiny pockets of air in the spinal canal (yellow arrow) and pneumomediastinum (red arrow). CT, computed tomography.

complaints or any clinical symptoms for novel coronavirus disease 2019 (COVID-19). The RT-PCR test turned out to be positive for COVID-19 and he was managed with supportive care in the isolation ward of the institution.

On the day 4 of admission with complaints of abdominal pain, he underwent contrast-enhanced $\mathrm{CT}$ abdomen which revealed few tiny pockets of air in the spinal canal (-Fig. 1). Our patient had no history of pulmonary disease, pneumothorax in the past, or tobacco use. To further evaluate the cause for the same CT of neck and thorax was done which showed pneumomediastinum, extensive subcutaneous emphysema, and PR. CT findings did not show any evidence of esophageal rupture or findings contributing toward the cause, labeling our diagnosis as spontaneous pneumomediastinum, and PR (-Fig. 2). The patient was asymptomatic related to these findings and his vitals were stable. No interventions were performed attributable to the cause and he was managed conservatively under the constant supervision of a respiratory physician. The patient underwent follow-up non contrast CT chest on days 7 and 10 which showed gradual resolution of the free mediastinal and intra spinal air but development of new nodular infiltrate in right lung parenchyma which was likely infective etiology
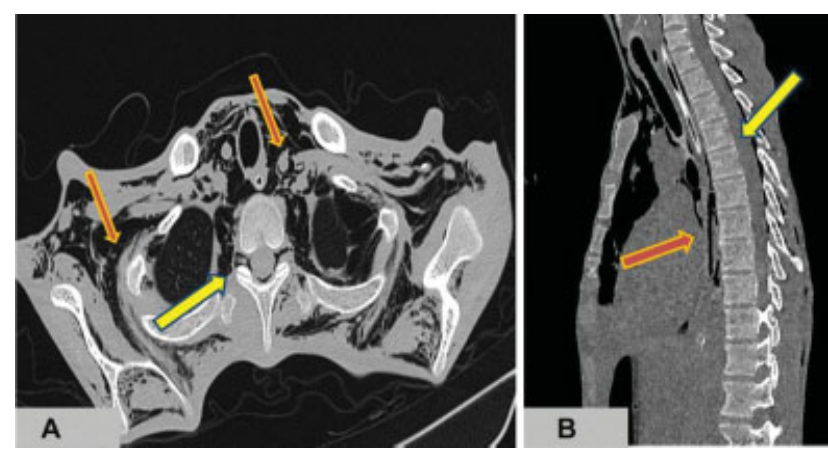

Fig. 2 (A) Axial CT image shows few tiny pockets of air in the spinal canal (yellow arrow) and extensive pneumomediastinum (red arrow). (B) Sagittal CT reconstruction to demonstrate the intra-spinal air (yellow arrow). CT, computed tomography.

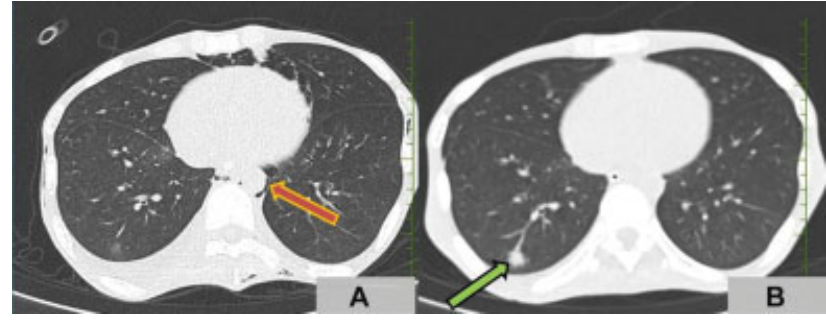

Fig. 3 (A) Axial CT image done on the day 7 of admission to show residual pneumomediastinum. (red arrow). (B) Axial CT image done on the day 10 of admission shows new nodular infiltrate in right lower lung parenchyma (green arrow). CT, computed tomography.

(-Fig. 3) Subsequently, the patient developed acute respiratory distress syndrome and was put on mechanical ventilator but expired on the day 14 .

\section{Discussion}

PR was first reported by Gordon and Hardman ${ }^{7}$ and the term "pneumorrhachis" was first coined by Newbold et al in $1982 .{ }^{8}$ PR is classified as an external (subdural/subarachnoid) or internal type (intraspinal epidural). The internal type needs more attention, as it is associated with major spinal trauma. ${ }^{1,3}$ In the direct route of intraspinal entry, air may entrap due to a one-way air valve mechanism, and dissects indirectly between the paraspinal soft tissues into the epidural space of the spinal canal via the neural foramina and along the vascular and nerve root sheaths, thereby producing PR.

Apart from the traumatic or iatrogenic causes, cases have been reported in literature describing PR caused by violent coughing due to bronchial asthma or acute bronchitis, postcardiopulmonary resuscitation, airway obstruction due to foreign body aspiration, ${ }^{9}$ and after physical exertion or inhalational drug abuse of 3,4-methylenedioxymethamphetamine ("ecstasy") or marijuana. ${ }^{10}$ Song and Lee reported a case of PR secondary to pneumomediastinum that presented with progressive motor weakness and sensory deficits in the lower extremities. ${ }^{11}$ Spontaneous pneumomediastinum is rare in viral pneumonia. ${ }^{12}$ It has been reported in cases with severe acute respiratory syndrome-associated coronavirus pneumonia. ${ }^{12}$ Although the exact mechanism is unknown, increased alveolar pressure and diffuse alveolar injury in severe COVID-19 pneumonia may make the alveoli prone to rupture, especially as patients often have repeated bouts of pronounced cough. Few reports on spontaneous pneumomediastinum from COVID-19 in the setting of noninvasive ventilation have been reported, although some cases have been complicated by pneumothorax. The development of pneumomediastinum in COVID-19 infection is considered a possible indicator of worsening disease ${ }^{1}$ but our patient fortunately survived.

The management of patients with PR must be decided on an individual basis and frequently requires a multidisciplinary regime. If intraspinal air is present, especially if caused by trauma, the spine physician must consider PR as an initial sign of potentially associated, hidden, and severe diseases or injuries. The role of a radiologist necessarily is to 
differentiate the internal and external types of PR and to search for other pathological conditions during the evaluation of a patient and should further investigate the underlying disease or whole extent of injuries.

\section{Conclusion}

Thus, evaluation of PR could be a diagnostic challenge. Although PR per se usually is self-limiting and without further therapeutic consequences, prompt recognition of the underlying cause is essential. So, noncontrast CT would suffice for confirming the etiology and to see the extent of involvement. The attending spine specialist must carefully evaluate the associated pathologies leading to PR to enable adequate therapy.

\section{Ethical Approval}

Institutional Review Board approval was obtained for this study.

\section{Funding Source}

None.

\section{Conflicts of Interest}

None declared.

\section{References}

1 Martins L, Dionísio P, Moreira S, Manique A, Correia I, Bárbara C. An unusual association in an uncommon disease: two cases of spontaneous pneumomediastinum associated with pneumorrhachis. Case Rep. Pulmonol 2016;2016;5092157
2 Oertel MF, Korinth MC, Reinges MHT, Krings T, Terbeck S, Gilsbach JM. Pathogenesis, diagnosis and management of pneumorrhachis. Eur Spine J 2006;15(5, suppl 5)636-643

3 Yaginuma K, Watanabe M, Saito Y, et al. Pneumorrhachis in children: a report of two cases and review of the literature. Radiol Case Rep 2019;14(11):1325-1329

4 Tsuji H, Takazakura E, Terada Y, Makino H, Yasuda A, Oiko Y. CT demonstration of spinal epidural emphysema complicating bronchial asthma and violent coughing. J Comput Assist Tomogr 1989; 13(01):38-39

5 Chiba Y, Kakuta H. Massive subcutaneous emphysema, pneumomediastinum, and spinal epidural emphysema as complications of violent coughing: a case report. Auris Nasus Larynx 1995;22 (03):205-208

6 Coniglio M, De Santis M, Pizzi G, Francioni F, Ricci P. Pneumorachis associated with spontaneous pneumomediastinum. A case report [in Italian]. Radiol Med (Torino) 1997;94(05):531-532

7 Gordon IJ, Hardman DR. The traumatic pneumomyelogram. A previously undescribed entity. Neuroradiology 1977;13(02): 107-108

8 Newbold RG, Wiener MD, Vogler JB III, Martinez S. Traumatic pneumorrhachis. AJR Am J Roentgenol 1987;148(03):615-616

9 Dosios T, Fytas A, Zarifis G. Spontaneous epidural emphysema and pneumomediastinum. Eur J Cardiothorac Surg 2000;18(01): 123

10 Bernaerts A, Verniest T, Vanhoenacker F, Van den Brande P, Petré C, De Schepper AM. Pneumomediastinum and epidural pneumatosis after inhalation of "Ectasy". Eur Radiol 2003;13(03): 642-643

11 Song K-J, Lee K-B. Spontaneous extradural pneumorrhachis causing cervical myelopathy. Spine J 2009;9(02):e16-e18

12 López Vega JM, Parra Gordo ML, Diez Tascón A, Ossaba Vélez S. Pneumomediastinum and spontaneous pneumothorax as an extrapulmonary complication of COVID-19 disease. Emerg Radiol 2020;27(06):727-730 